\title{
Una solución analítica para la ecuación de difusión advección reacción por medio de la serie de Fourier
}

\author{
José R. Jiménez Zenteno \\ zenteno20jr@hotmail.com \\ Instituto Politécnico Nacional \\ IPN ESIA Unidad Ticóman, México \\ Recibido: Mayo 21, 2017 Aceptado: Diciembre 21, 2017
}

\begin{abstract}
Resumen. El presente trabajo presenta la ecuación de advección difusión reacción bajo condiciones de frontera mixta, para un caso de transporte de contaminación atmosférica el cual se soluciona por medio de la serie de Fourier dando una alternativa analítica al caso de solución numérica y con un forzamiento en un punto en específico. Se encontrará un coeficiente de Fourier el cual junto a una tasa de contaminante en función del tiempo se modelará en coordenadas cartesianas para la solución unidimensional. El software utilizado para la simulación es Matlab 2014, Se usarán dos ejemplos con dos tasas contaminantes, mostrando resultados óptimos dentro de las condiciones indicadas junto a las variables de velocidad de viento, coeficiente de difusión y reacción química.
\end{abstract}

Palabras clave: Serie de Fourier, función delta de Dirác, ecuación de advección difusión, separación de variable

Abstract. Abstract The present work presents the Advection Diffusion Reaction Equation under conditions of Mixed Border, for a case of Transport of Atmospheric Contamination which is solved by means of the Fourier Series giving an analytical alternative to the case of numerical solution and with a Forcing in a Point in specific. A Fourier Coefficient will be found which next to a Rate of Contaminant as a function of Time will be modeled in Cartesian Coordinates for the One-dimensional solution, The software used for the simulation is in Matlab 2014, Two examples will be used with two Rates Contaminants.Showing optimal results within the conditions indicated next to the variables of wind speed, Diffusion coefficient and chemical reaction.

KeyWords: Fourier series, Dirac Delta function, Diffusion Advection Equation, Variable Separation

\subsection{Introducción}

La ecuación de advección y difusión es una expresión muy importante dado a su amplio uso dentro de la modelación matemática en procesos de dispersión y difusión en el transporte de masa, calor, 
economía o un contaminante dentro de un medio poroso o atmosférico. Una sustancia contaminante contenida en el medio atmosférico se encuentra sujeta a diversos procesos físicos y químicos que influyen en su propagación y transformación. Algunos de estos procesos son: transporte por advección, sedimentación, difusión turbulenta y transformación por diversas reacciones químicas, además, debido a la complejidad de dichos procesos, la dispersión de cada sustancia que ha sido emitida a la atmósfera por fuentes fijas o móviles es un fenómeno tridimensional y no-estacionario. En lo que sigue se formula un modelo lineal que toma en cuenta estos procesos, y se supone que todos los coeficientes contenidos en las parametrizaciones respectivas son conocidos.

\subsection{Formulación de un modelo de dispersión de contaminantes atmosféricos}

Sea $\boldsymbol{D}=\boldsymbol{D} \times(0, H)$ un dominio acotado tridimensional de un espacio real en la atmosfera (conexo y simplemente conexo) con frontera abierta $\partial \boldsymbol{D}=S_{0} \cup S \cup S_{H}$. La frontera $H$ es la altura del espacio $\boldsymbol{D}$ tridimensional la cual es la unión de la superficie lateral cilíndrica $S$, la base o fondo $S_{0}$ y la cubierta $S_{H}$ en $z=H$ (figura 1.1). Se denota por $\mathbf{r}_{i}=\left(x_{i}, y_{i}, z_{i}\right) \in \mathbf{D}, i=1, \ldots, N$, los puntos donde se ubican las $N$ fuentes puntuales (con $N$ número natural de fuentes industriales) que emiten $K$ (número natural) sustancias contaminantes. Sustancia lo referimos a una clase particular de materia homogénea cuya composición es fija y químicamente definida como por ejemplo ozono. Se supone que la velocidad con la que decae cada una de estas sustancias es proporcional a su concentración en la atmósfera o dentro del dominio de $\boldsymbol{D}$. La tasa de emisión que corresponde a cada fuente puntual se denota como $q_{i}(t), i=1, \ldots, N$. Finalmente, se denotan con $\varphi_{k}(\boldsymbol{r}, t)$ a la concentración del $k$-ésimo contaminante dentro de la región $D$ en el punto $r$ al tiempo $t$.

Es importante destacar que se supone aquí que la región de estudio $\boldsymbol{D}$ contiene a todas las fuentes puntuales de emisión de los contaminantes, y por lo tanto, no habrá contribuciones externas a la polución dentro del dominio $\boldsymbol{D}$ del espacio atmosférico. Tomando en cuenta esto en el balance de masa, la propagación de los contaminantes en la atmósfera se puede describir a través del siguiente sistema de ecuaciones ([3, Parra-Guevara, D.,Skiba, Y. N., 2003]).

El término $\nabla \cdot \varphi_{k}^{s}$ describe el cambio en la concentración de la sustancia observada (p. ej. algún tipo de partículas), por unidad de tiempo, y debido a la sedimentación; tal proceso está caracterizado por la velocidad constante de sedimentación $v_{k}^{s}>0$, donde $s$ es solo un subíndice indicando esa velocidad de sedimentación.

$$
\begin{gathered}
\frac{\partial \varphi_{k}}{\partial t}+U \cdot \nabla \varphi_{k}+\nabla \cdot \varphi_{k} s-\nabla \cdot\left(\mu \nabla \varphi_{k}\right)-\frac{\partial}{\partial z}\left(\mu_{z} \frac{\partial \varphi_{k}}{\partial z}\right)+\sigma_{k} \varphi_{k}=f_{k}(r, t) \quad \text { en } \boldsymbol{D} \times(0, T) \\
\varphi_{k}(r, 0)=\varphi_{k}^{0}(r) \text { en } \boldsymbol{D} \\
\varphi_{k}^{s}=-v_{k}^{s} \varphi_{k} e_{3} \text { en } \boldsymbol{D} \\
\mu_{z} \frac{\partial \varphi_{k}}{\partial z}-U_{n} \varphi_{k}=-v_{k}^{s} \varphi_{k} \text { en } S_{H}^{-}
\end{gathered}
$$




$$
\begin{gathered}
\mu_{z} \frac{\partial \varphi_{k}}{\partial z}=-v_{k}^{s} \varphi_{k} \text { en } S_{H}^{+} \\
\mu \nabla \varphi_{k} \cdot \boldsymbol{n}-U_{n} \varphi_{k}=0 \text { en } S^{-} \\
\mu \nabla \varphi_{k} \cdot \boldsymbol{n}=0 \text { en } S^{+} \\
\mu^{*} \nabla \varphi_{k} \cdot \boldsymbol{n}=0 \text { en } S_{0} \\
\nabla \cdot U=0 \text { en } D
\end{gathered}
$$

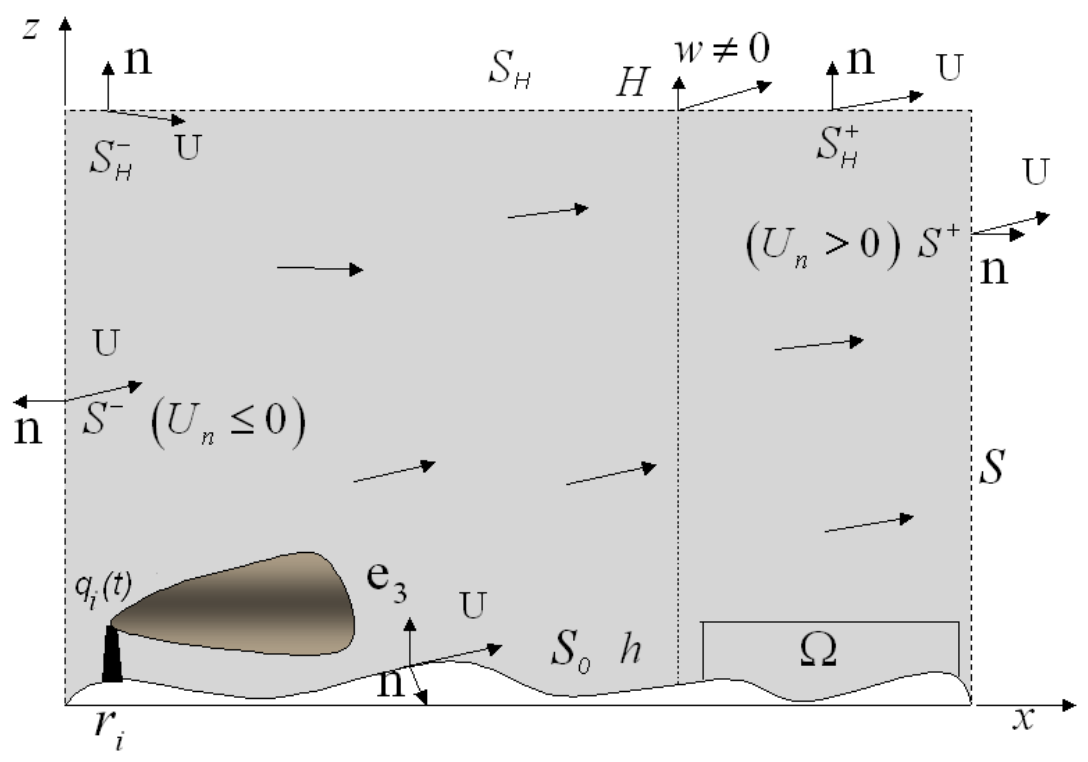

Figura 1.1: Proyección en el plano $x-z$ del modelo conceptual de dispersión [1, D. Parra-Guevara y Y. Skiba, 2016]

Donde $\mathbf{U}(\boldsymbol{r}, t)=(u, v, w)$ denota la velocidad del viento en la región $\boldsymbol{D}$ y se supone que cumple la ecuación de continuidad, la ecuación 1.9. Además, $\sigma_{k}=\sigma_{k}(r, t) \geq 0$ es el coeficiente de reacción química de la $k$-ésimasustancia contaminante el cual involucra la relación entre la radiación solar, interacción química entre las sustancias contaminantes con su entorno ambiental como la temperatura o humedad. Y los coeficientes $\mu=\mu(\mathbf{r}, t)>0$ y $\mu^{*}=\mu(\boldsymbol{r}, t)>0$ denotan a los tensores de difusión turbulenta, es cualquier proceso de difusión por el cual las sustancias se mezclan en la atmósfera o en cualquier sistema de fluido debido al movimiento turbulento del viento y donde $\varphi$ es la concentración del contaminante.

$$
\begin{gathered}
\mu=\left[\begin{array}{cc}
\mu_{x}(\boldsymbol{r}, t) & 0 \\
0 & \mu_{y}(\boldsymbol{r}, t)
\end{array}\right] \\
\mu^{*}=\left[\begin{array}{ccc}
\mu_{x}(\boldsymbol{r}, t) & 0 & 0 \\
0 & \mu_{y}(\boldsymbol{r}, t) & 0 \\
0 & 0 & \mu_{z}(\boldsymbol{r}, t)
\end{array}\right]
\end{gathered}
$$


Respectivamente, y $f_{k}(\boldsymbol{r}, t)$ es el forzamiento formado por las tasas de emisión para el $k$-ésimo contaminante, el cual es $q_{i k}$, la taza de emisión modelada o medida dentro de un período de tiempo por ejemplo a 24 horas, los $k$ contaminantes emitidos pueden ser desde una sola sustancia de contaminante como ejemplo Ozono troposférico [O3] o los que estén presentes dentro del dominio $\boldsymbol{D}$ definido anteriormente, con unidades de $[\mu \mathrm{g} / \mathrm{m} 3]$ :

$$
f_{k}(\boldsymbol{r}, t)=\sum_{i=1}^{\infty} q_{i k}(t) \delta\left(\boldsymbol{r}-\boldsymbol{r}_{i}\right)
$$

Donde $\delta\left(\boldsymbol{r}-\boldsymbol{r}_{i}\right)$ es la función delta de Dirác centrada en la posición de la $i$-ésima fuente puntual. Hay que notar que la tasa de emisión de cada fuente es la suma de las tasas para cada contaminante, es decir,

$$
q_{i}(t)=\sum_{k=1}^{K} q_{i k}(t)
$$

La ecuación 1.2 define a $\varphi_{k}^{0}$ como la distribución espacial de la $k$-ésima sustancia contaminante al tiempo $t=0$ sobre $D$,es decir, $\varphi_{k}^{0}$ es el residuo del $k$-ésimo contaminante en la atmósfera que dejó la actividad industrial en un intervalo de tiempo pasado (por ejemplo durante el día anterior).

La frontera $S$ se ha dividido en cinco partes, dos para el flujo horizontal, es decir, $S^{+}$se define como los puntos de $S$ tales que $U_{n}=\mathbf{U} \cdot \boldsymbol{n}>0$, donde $\boldsymbol{n}$ es el vector normal exterior en la dirección x, y $S^{-}$ se define como el complemento $\left(U_{n}=\mathbf{U} \cdot \boldsymbol{n} \leq 0\right)$. Dos para la frontera superior: $S_{H}^{-}$es la parte de la frontera donde la sedimentación es el resultado de la difusión menos la Advección (ecuación 1.4), y $S_{H}^{+}$ que implica que la sedimentación es igual a la difusión (ecuación 1.5). Por último, en $S_{0}$, que es la frontera inferior, se tiene que la difusión es cero debido a que el flujo es tangente a la superficie irregular (ecuación 1.8). La condición de frontera 1.6 establece que cuando el viento ingresa a la región Del flujo total del contaminante, tomando en cuenta difusión y advección, es igual a cero, por lo cual, en $S^{-}$no hay salida o entrada de la especie contaminante. La condición de frontera 1.7 establece que cuando el viento sale de la región $\boldsymbol{D}$ se desprecia el flujo difusivo turbulento es un régimen caracterizado por baja difusión de momento, alta convección y cambios espacio-temporales rápidos de presión y velocidad.En comparación con el flujo advectivo es el transporte de una sustancia contaminante por efecto del viento, por lo tanto, la salida de la sustancia contaminante sólo es por advección ([2, Marchuk, 1986 ], [7, Skiba, 1993 ] y [8, Skiba, Y. N., Parra-Guevara D, 2000]).

Para concluir la definición del modelo anterior el significado de la ecuación de continuidad, que cierra dicho modelo de dispersión, la cual es una condición de incompresibilidad, ecuación 1.9

\subsection{Ecuaciones gobernantes en casos unidimensionales}

Sistema Principal a resolver

$$
\frac{\partial \varphi}{\partial t}+u \frac{\partial \varphi}{\partial x}-\mu \frac{\partial^{2} \varphi}{\partial x^{2}}+\sigma \varphi=q(t) \delta\left(x-x_{0}\right)
$$




$$
\begin{gathered}
\varphi(x, 0)=0,0<x<l \\
\mu \frac{\partial \varphi(l, t)}{\partial x}=0, \quad t>0 \\
\mu \frac{\partial \varphi(0, t)}{\partial x}-u \varphi(0, t)=0, \sigma>0
\end{gathered}
$$

Las otras direcciones dentro del dominio del espacio tridimensional $\boldsymbol{D}$

$$
\begin{gathered}
\frac{\partial \varphi}{\partial t}+v \frac{\partial \varphi}{\partial y}-\mu \frac{\partial^{2} \varphi}{\partial y^{2}}+\sigma \varphi=q(t) \delta\left(y-y_{0}\right) \\
\varphi(y, 0)=0, \quad 0<y<l \\
\mu \frac{\partial \varphi(l, t)}{\partial y}=0, \quad t>0 \\
\frac{\partial \varphi(0, t)}{\partial y}-v \varphi(0, t)=0, \quad \sigma>0 \\
\frac{\partial \varphi}{\partial t}+\left(u-V_{k}\right) \frac{\partial \varphi}{\partial z}-\left(\mu_{z}\right) \frac{\partial^{2} \varphi}{\partial z^{2}}+\sigma \varphi=q(t) \delta\left(z-z_{0}\right) \\
\varphi(z, 0)=0,0<z<l \\
\frac{\partial \varphi(0, t)}{\partial x}+\frac{V_{k}}{\mu_{z}} \varphi(0, t)=0, \quad t>0 \\
\frac{\partial \varphi(l, t)}{\partial x}-\frac{2 w}{\mu_{z}} \varphi(l, t)=0, \quad \sigma>0
\end{gathered}
$$

\subsubsection{Solución en la dirección unidimensional $x$}

$$
\varphi(x, t)=w(x, t) e^{r x-s t}, 0<x<l
$$

$\mathrm{Al}$ aplicar la transformación obtenemos lo siguiente, donde $D=\mu$ :

$$
r=\frac{v}{2 D} \quad \mathrm{y} \quad s=\left(\frac{v^{2}}{4 D}+\sigma\right)
$$

Y la ecuación reducida es:

$$
\frac{\partial w}{\partial t}=D \frac{\partial^{2} w}{\partial x^{2}}+e^{s t} F(x, t), \quad \text { con } F(x, t)=\gamma e^{-r x}
$$

Donde gamma es 


$$
\gamma=q(t) \delta\left(x-x_{0}\right)
$$

Aplicando a las condiciones de frontera se tiene que:

$$
\begin{gathered}
w(x, 0)=0 \\
w_{x}(0, t)-r w(0, t)=0 \\
w_{x}(l, t)+r w(l, t)=0
\end{gathered}
$$

Nota: Al transformar la condición de frontera de

$$
\mu \frac{\partial \varphi(l, t)}{\partial x}=0, \quad t>0
$$

con $w\left(x, t_{0}\right)$ se obtiene la misma transformación de

$$
w_{x}(l, t)+r w(l, t)=0
$$

Así ahora resolviendo la ecuación en forma homogénea del sistema a exponer

$$
\begin{gathered}
\frac{\partial w}{\partial t}=D \frac{\partial^{2} w}{\partial x^{2}} \\
w(x, 0)=0 \\
w_{x}(0, t)-r w(0, t)=0 \\
w_{x}(l, t)+r w(l, t)=0
\end{gathered}
$$

Ahora usando la separación de variables $w=\phi(x) T(t)$ y el Eigenvalor de $-\lambda_{n}^{2}<0$, las demás soluciones son triviales y la solución es compleja

$$
\begin{aligned}
& \phi_{x x}+\lambda_{n}^{2} \phi(x)=0 \\
& \phi(0)_{x}-r \phi(0)=0 \\
& \phi(l)_{x}+r \phi(l)=0
\end{aligned}
$$

Con solución de

$$
\phi_{n}(x)=\cos \left(x \lambda_{n}\right)+\frac{r}{\lambda_{n}} \operatorname{sen}\left(x \lambda_{n}\right) C_{2}=\frac{r}{\lambda_{n}} C_{1} \text { con } C_{1}=1
$$


La trascendente es y haciendo las sustituciones correspondientes en $r$

$$
\tan \left(l \lambda_{n}\right)=-\frac{4 \mu u \lambda_{n}}{u^{2}-4 \mu^{2} \lambda_{n}^{2}}
$$

La ecuación en $T$, con la condición temporal en $t=0$,

$$
\begin{gathered}
\frac{d T_{n}}{d t}+D \lambda^{2} T_{n}=0 \\
T(0)=0
\end{gathered}
$$

Ahora por el método de expansión de funciones propias (ver [6]), se obtiene:

$$
w(x, t)=\sum_{n=1}^{\infty} \phi_{n}(x) T_{n}(t)
$$

La solución general en $T$ es:

$$
T_{n}(t)=\frac{e^{-D \lambda_{n} t}}{\int_{0}^{l} \phi_{n}(x)^{2} d x}\left(\int_{0}^{t} e^{\left(s+D \lambda_{n}^{2}\right) t} \int_{0}^{l} F(x, \zeta) \phi_{n}(x) d x d \zeta\right)
$$

Ahora se tiene la serie de Fourier como

$$
w(x, t)=\sum_{n=1}^{\infty} \phi_{n}(x) \frac{e^{-D \lambda_{n}^{2} t}}{\int_{0}^{l} \phi_{n}(x)^{2} d x}\left(\int_{0}^{t} e^{\left(s+D \lambda_{n}^{2}\right) t} \int_{0}^{l} F(x, \zeta) \phi_{n}(x) d x d \zeta\right)
$$

Y recordando que se tiene ?? Multiplicando se obtiene la solución general

$$
\varphi(x, t)=\sum_{n=1}^{\infty} \phi_{n}(x) \frac{e^{r x-\left(s+D \lambda_{n}^{2}\right) t}}{\int_{0}^{l} \phi_{n}(x)^{2} d x}\left(\int_{0}^{t} e^{\left(s+D \lambda_{n}^{2}\right) \zeta} \int_{0}^{l} F(x, \zeta) \phi_{n}(x) d x d \zeta\right)
$$

Ahora con

$$
\begin{gathered}
F(x, \zeta)=F(x, t)=\gamma e^{-r x} \\
\gamma=q(t) \delta\left(x-x_{0}\right)
\end{gathered}
$$

obtengamos los coeficientes de Fourier, viendo en $T_{n}(t)$

$$
\sum_{n=1}^{\infty}\left[\frac{d T_{n}(t)}{d t}+D \lambda_{n}^{2} T_{n}(t)\right] \phi_{n}(x)=F(x, t) \sum_{n=1}^{\infty}\left[\frac{d T_{n}(t)}{d t}+D \lambda_{n}^{2} T_{n}(t)\right] \phi_{n}(x)=F(x, t)
$$

Y $F(r, t)$ admite la siguiente forma de producto de dos funciones 


$$
F(x, t)=F(t) \phi_{n}(x)
$$

Así, sustituyendo nos da

$$
\sum_{n=1}^{\infty}\left[\frac{d T_{n}(t)}{d t}+D \lambda_{n}^{2} T_{n}(t)\right]=F(t)
$$

La solución temporal homogénea es:

$$
\begin{gathered}
T_{n}(t)=T e^{-\lambda_{n}{ }^{2} D t} \\
{\left[-\lambda_{n}^{2} D T_{n}(t) e^{-\lambda_{n}{ }^{2} D t}+\frac{d T_{n}(t)}{d t} e^{-\lambda_{n}^{2} D t}\right]+\lambda_{n}^{2} D T_{n}(t) e^{-\lambda_{n}^{2} D t}=F(t)} \\
F(t)=\frac{d T_{n}(t)}{d t} e^{-\lambda_{n}^{2} D t} \\
\frac{d T_{n}(t)}{d t}=F(t) e^{\lambda_{n}^{2} D t} \\
T_{n}(t)=\int_{0}^{t} F(\tau) e^{-\lambda_{n}^{2} D(t-\tau)} d \tau
\end{gathered}
$$

Finalmente sustituimos en $F(r, t)$ el cual es una serie de Fourier

$$
\begin{gathered}
F(x, t)=F(t) \phi_{n}(x)=F(t)\left(\cos \left(x \lambda_{n}\right)+\frac{r}{\lambda_{n}} \operatorname{sen}\left(x \lambda_{n}\right)\right) \\
\int_{0}^{l} F(x, t) \cos \left(x \lambda_{n}\right) d x=F(t) \int_{0}^{l} \cos \left(x \lambda_{n}\right)^{2} d x \\
\frac{\lambda_{n}}{r} \int_{0}^{l} F(x, t) \operatorname{sen}\left(x \lambda_{n}\right) d x=F(t) \int_{0}^{l} \operatorname{sen}\left(x \lambda_{n}\right)^{2} d x
\end{gathered}
$$

Sustituyendo finalmente

$$
\begin{gathered}
w(x, t)=\sum_{n=1}^{\infty} \phi_{n}(x) T_{n}(t) \\
w(x, t)=\sum_{n=1}^{\infty} \phi_{n}(x)\left[\int_{0}^{t} F(\tau) e^{-\lambda_{n}^{2} D(t-\tau)} d \tau\right] \\
w(x, t)=\sum_{n=1}^{\infty} \phi_{n}(x)\left[\int_{0}^{t} e^{-\lambda_{n}^{2} D(t-\tau)}\left(\frac{\int_{0}^{l} F(x, t) \cos \left(x \lambda_{n}\right) d x}{\int_{0}^{l} \cos \left(x \lambda_{n}\right)^{2} d x}+\frac{\frac{\lambda_{n}}{r} \int_{0}^{l} F(x, t) \operatorname{sen}\left(x \lambda_{n}\right) d x}{\int_{0}^{l} \operatorname{sen}\left(x \lambda_{n}\right)^{2} d x}\right) d \tau\right]
\end{gathered}
$$


Así finalmente obtenemos los coeficientes en función de la delta de Dirác, pero hay un problema de convergencia el cual no nos ayuda para solución, aplicando la serie de Fejér no logra dar una convergencia, as'1 que tenemos que usar la forma de serie de la delta y así obtendremos un nuevo coeficiente de Fourier el cual nos ayudara, notar que el signo entre ambos coeficientes da el sentido de la Tasa por encima o por debajo del eje temporal $t$

$$
d_{n}=\frac{\cos \left(x_{0} \lambda_{n}\right)}{\left(\frac{1}{2}+\frac{\operatorname{sen}\left(2 \lambda_{n}\right)}{4 \lambda_{n}}\right)}+\frac{\frac{\lambda_{n}}{r} \operatorname{sen}\left(x_{0} \lambda_{n}\right)}{\left(\frac{1}{2}-\frac{\operatorname{sen}\left(2 \lambda_{n}\right)}{4 \lambda_{n}}\right)}
$$

Forzamiento con función delta modificada

Ahora obteniendo los coeficientes de Fourier con la delta modificada. Sea ahora, con la delta en forma de serie

$$
\delta\left(x-x_{0}\right)=\left[\frac{1}{2}+\frac{1}{L} \sum_{n=1}^{\infty} \cos \left(n\left(x-x_{0}\right)\right)\right]
$$

Con $L=1$

Nos quedará como

$$
q(t)\left[\delta\left(x-x_{0}\right)\right]=\sum_{n=1}^{\infty} F(t) \phi_{n}(x)
$$

El cual es para la obtención de los coeficientes de Fourier en la forma en que sacamos los coeficientes en 1.56

Coeficientes de Fourier con la delta modificada

$$
\begin{gathered}
d_{n}=\frac{0.5+\cos \left(x_{0} \lambda_{n}\right)\left(0.5\left(1+\frac{\sin \left(2 \lambda_{n}\right)}{2 \lambda}\right)\right)+\operatorname{sen}\left(x_{0} \lambda_{n}\right)\left(\frac{\operatorname{sen}\left(\lambda_{n}\right)^{2}}{2 \lambda_{n}}\right)}{0.5\left(1+\frac{\sin \left(2 \lambda_{n}\right)}{2 \lambda_{n}}\right)} \\
+\frac{0.5+\cos \left(x_{0} \lambda_{n}\right)\left(\frac{\operatorname{sen}\left(\lambda_{n}\right)^{2}}{2 \lambda_{n}}\right)+\operatorname{sen}\left(x_{0} \lambda_{n}\right)\left(0.5\left(1-\frac{\sin \left(2 \lambda_{n}\right)}{2 \lambda_{n}}\right)\right)}{\frac{r}{2 \lambda_{n}}\left(1-\frac{\sin \left(2 \lambda_{n}\right)}{2 \lambda_{n}}\right)}
\end{gathered}
$$

Así tenemos la solución con los coeficientes de Fourier, siendo esta una alternativa a la solución numérica por diferencia finita por separación de operadores ([4]).

\subsubsection{Pruebas de cada coeficiente de Fourier}

Ahora veamos ejemplos con fuentes de emisión usando Matlab 2014, los resultados con las propiedades de la delta de Dirá con los coeficientes como: 


$$
d_{n}=\frac{\cos \left(x_{0} \lambda_{n}\right)}{\left(\frac{1}{2}+\frac{\operatorname{sen}\left(2 \lambda_{n}\right)}{4 \lambda_{n}}\right)}+\frac{\frac{\lambda_{n}}{r} \operatorname{sen}\left(x_{0} \lambda_{n}\right)}{\left(\frac{1}{2}-\frac{\operatorname{sen}\left(2 \lambda_{n}\right)}{4 \lambda_{n}}\right)}
$$

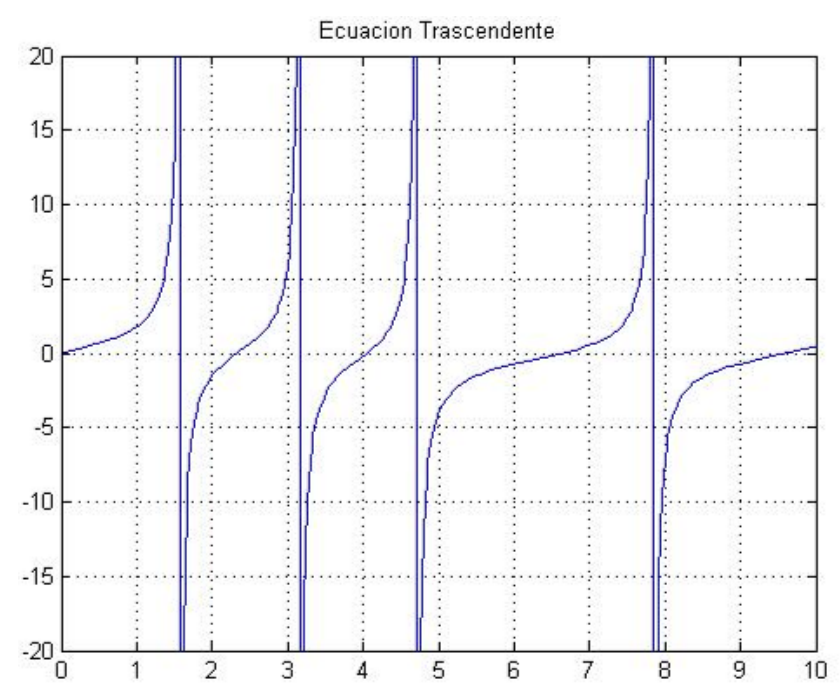

Figura 1.2: Ecuación Trascendente

Las soluciones de la ecuación trascendente son los eigenvalores dados por la ecuación 1.42

\begin{tabular}{c}
\hline-1.98635037748832 \\
3.67319440630425 \\
6.58462004256418 \\
9.63168463569188 \\
12.7232407841313 \\
15.8341053693324 \\
18.9549714108416 \\
22.0816596359426 \\
25.2120268885509 \\
28.3448641495999 \\
31.4794387120098 \\
34.6152810748294 \\
37.7520766759718 \\
40.8896069332367 \\
44.0277152732461 \\
\hline
\end{tabular}

Ahora los coeficientes de Fourier con las propiedades de la delta de Dirác, se observa que no convergen 


\begin{tabular}{c}
\hline-1147.1563872571 \\
-2424.0528217396 \\
-4153.0970002009 \\
285.1714593412 \\
8764.1333681701 \\
1093.2856260382 \\
389.1372909394 \\
-1486.8841077235 \\
-1793.0180777803
\end{tabular}

Tasa Pulso.

Velocidad del viento $u$, coeficiente de difusión $\mu$ y coeficiente de reacción química $\sigma: u=0.1 \mathrm{~m} / \mathrm{s}$, $\mu=0.05 \mathrm{~m}^{2} / \mathrm{s}, \sigma=0.1$ y $x_{0}=0.2 \mathrm{~km}$ para ambas tasas mostradas abajo

$$
q(t)=\left\{\begin{array}{c}
1002 \leq x \leq 4 \\
0 x<2 y x>4
\end{array}\right\}
$$

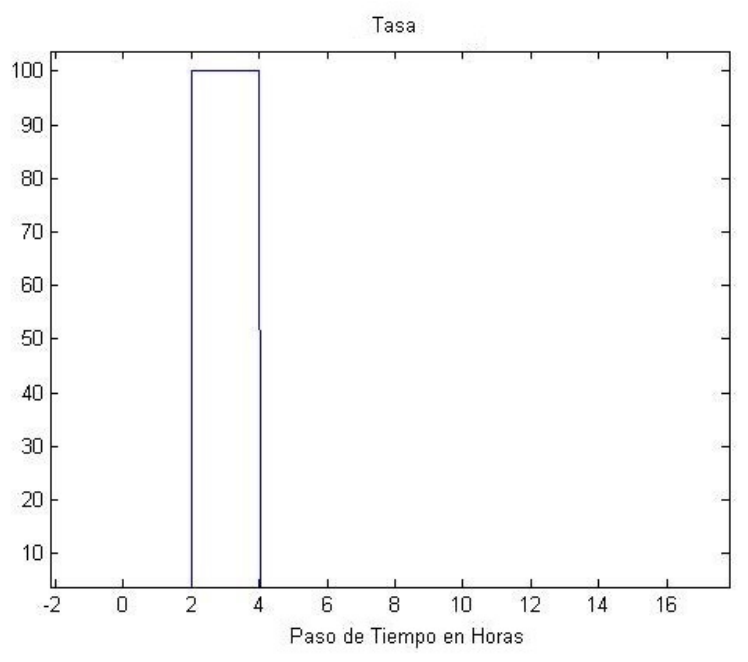

Figura 1.3: Tasa Pulso Cuadrado

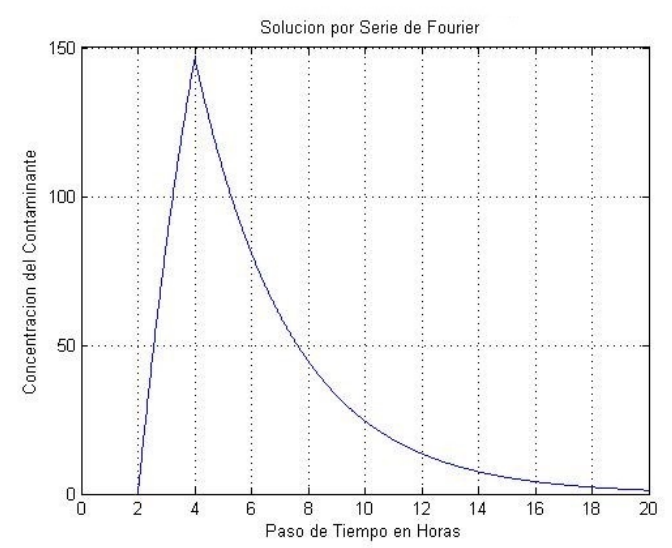

Figura 1.4: Tasa Pulso Cuadrado 
No converge la solución, la tasa que se obtiene es con una sobreestimación de la concentración aproximada.

Tasa seno.

$$
q(t)=\left\{\begin{array}{lc}
100 t^{2} e^{-0.5 t} \sin ^{2}(t), & 0<t<4 \pi \\
0 & \text { de otra forma }
\end{array}\right\}
$$

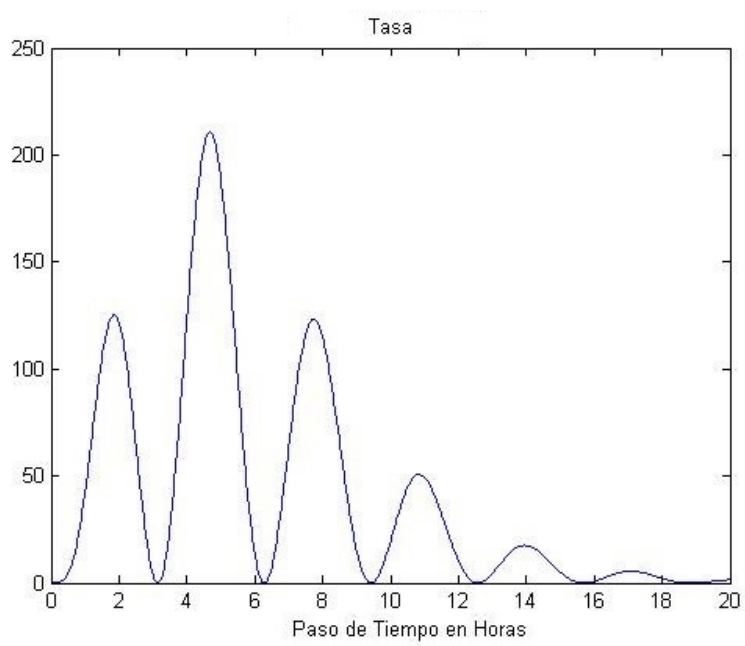

Figura 1.5: Tasa seno

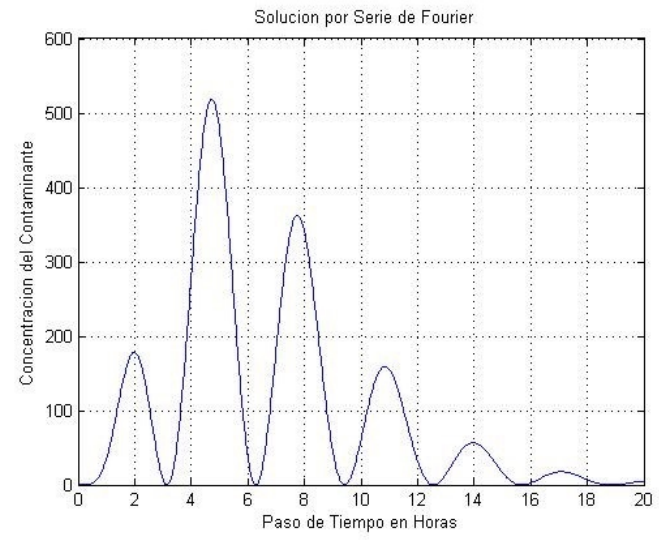

Figura 1.6: Tasa seno

Obteniendo los coeficientes de Fourier con la delta modificada.

Los coeficientes de Fourier con la delta modificada usando 1.3.1 son convergentes negativamente. 


$$
\begin{aligned}
& -35.9296480491164 \\
& -3083.28725361635 \\
& -4900.46631915789 \\
& -3770.97517245334 \\
& -710.083025985008 \\
& -873.014581624824 \\
& -7431.88390813713 \\
& -16332.4418665503 \\
& -19110.0362029934 \\
& -12128.0823475606 \\
& -2247.78492895684 \\
& -1293.08765599530 \\
& -13561.4891488819 \\
& -29508.1020204595 \\
& -33866.4135057151 \\
& -21462.9871559900 \\
& -4386.90384516323
\end{aligned}
$$

Tasa Pulso.

Velocidad del viento $u$, coeficiente de difusión $\mu$ y coeficiente de reacción química $\sigma: u=0.1 \mathrm{~m} / \mathrm{s}$, $\mu=0.05 \mathrm{~m}^{2} / \mathrm{s}, \sigma=0.1$ y $x_{0}=0.2 \mathrm{~km}$

Solución por serie de Fourier usando los coeficientes de Fourier con la delta modificada:

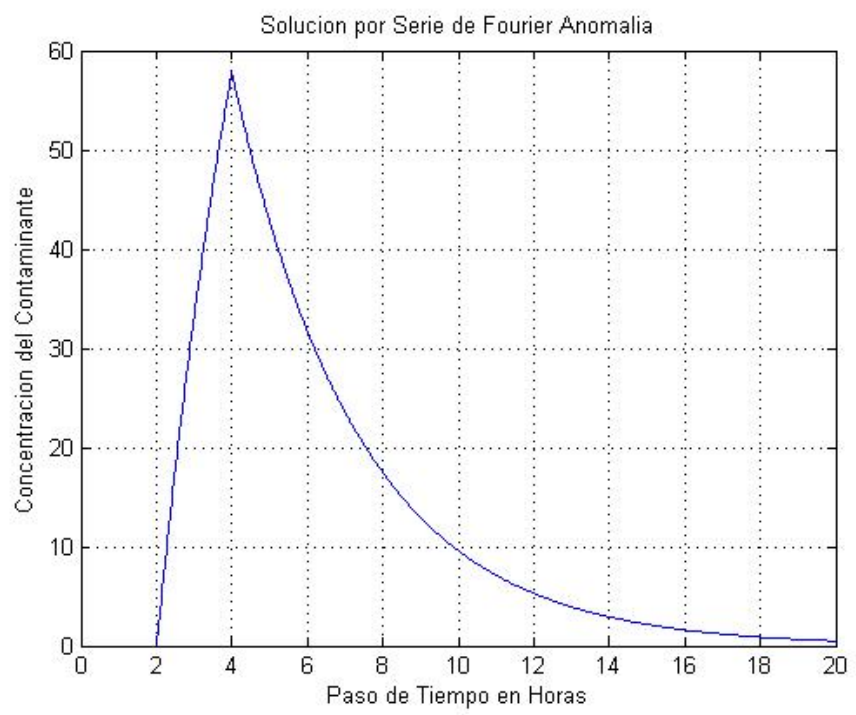

Figura 1.7: Tasa Pulso con la delta modificada

Tasa Seno

Velocidad del viento $u$, coeficiente de difusión $\mu$ y coeficiente de reacción química $\sigma$ $u=3.87 \mathrm{~m} / \mathrm{s} \mu=0.40 \mathrm{~m}^{2} / \mathrm{s} \sigma=0.0, x_{0}=0.2 \mathrm{~km}$ de la fuente. 
Solución por serie de Fourier usando los coeficientes de Fourier con la delta modificada

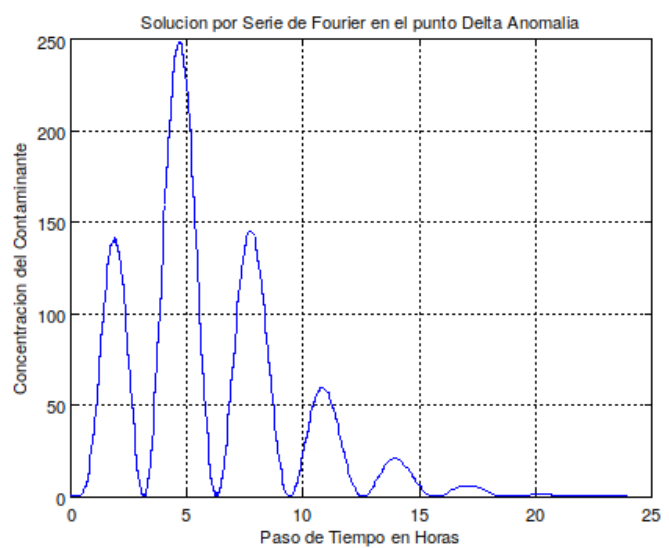

Figura 1.8: Tasa Seno con la delta modificada

Converge la solución, la tasa obtenida esta con los efectos de los términos de difusión y advección de la concentración aproximada observada.

\subsection{Conclusiones}

Los resultados obtenidos muestran una dependencia del punto de emisión de las tasas en que se originó la emisión observada, las soluciones son muy susceptibles a las condiciones entre $x_{0}, u$ y $\sigma$, en el cual el punto $x_{0}$ debe de estar de preferencia lo más cerca de la medición de la tasa de emisión $q(t)$, otro punto que aclarar es el signo que está entre ambos coeficientes de la cual la fórmula de Euler o fórmula de Euler-Cotes (http://mathworld. wolfram.com/EulerFormula.html ) nos da una respuesta: $e^{i x}=\cos x+i \operatorname{sen} y$ tal y como en la solución se obtiene el valor en su forma compleja y para obtener el coeficiente de $\frac{r}{\lambda_{n}}$ tiene una forma cuadrática debido al eigenvalor propuesto, así toma la forma $i^{2}$, en la solución tomando la forma en el sentido del signo ya que este le da el sentido positivo o negativo de la función en la solución final en el eje temporal o sea $t$ (tiempo).

Esta es una solución propuesta a la alternativa numérica, la función delta genera problemas con la propiedad general al obtener los coeficientes de Fourier en la forma convencional, pero al modificarla mejora este aspecto; en la solución numérica no se obtiene este problema dado que solo es un punto o posición en dicho intervalo espacial dentro del mallado en la discretización del sistema unidimensional.

\section{Bibliografía}

[1] David Parra-Guevara y Yuri N. Skiba. "Recuperación de la Tasa de Emisión de una Fuente Contaminante: Análisis de la Existencia, la Unicidad y la Estabilidad de las Soluciones". Información Tecnológica Vol. 27(5), 251-262 (2016) 
[2] Marchuk, G. I. (1986). Mathematical Models in Environmental Problems. Elsevier, New York.

[3] Parra-Guevara, D. \& Skiba, Y. N. (2003). "Elements of the matematical modelling in the control of pollutants emissions". Ecological Modelling, 167, 263-275.

[4] Parra-Guevara, D, Skiba, Yu.N., \& Zenteno J.R (2005). Introducción a los Métodos de Dispersión y Control de Contaminantes. la UNAM. México. https://www.researchgate.net/publication/ 236164913_Introduccion_a_los_Metodos_de_Dispersion_y_Control_de_Contaminantes

[5] Peter V. O’Neil Matemáticas Avanzadas para Ingeniería. Volumen 2 (1999) Cap. 18, 321-429

[6] José Darío Sánchez Hernández. Elementos de Ecuaciones Diferenciales Parciales (1984), Cap 6.9, 49-50

[7] Skiba, Y. N. (1993). Balanced and absolutely stable implicit schemes for the main and adjoint pollutant transport equations in limited area. Rev. Intern. Contamin. Ambient., 9, 39-51.

[8] Skiba, Y. N. \& Parra-Guevara, D. (2000). Industrial pollution transport. Part I: Formulation of the problem and air pollution estimates. Environmental Modeling and Assessment, 5, 169-175. 